\title{
Identification of a Prognostic Signature Based on the Expression of Genes Related to the Insulin Pathway in Early Breast Cancer
}

\author{
Alessandra Gennari ${ }^{a}$ Mariapia Sormani ${ }^{b}$ Matteo Puntoni ${ }^{c}$ Veronica Martini ${ }^{a}$ \\ Adriana Amaro ${ }^{d}$ Paolo Bruzzi ${ }^{\text {e }}$ Ulrich Pfeffer ${ }^{d}$ \\ a Division of Oncology, Department of Translational Medicine, University of Eastern Piedmont, Novara, Italy; \\ ${ }^{b}$ DISSAL, University of Genoa, Genoa, Italy; ${ }^{c}$ Clinical Trial Unit, Galliera Hospital Genoa, Genoa, Italy;

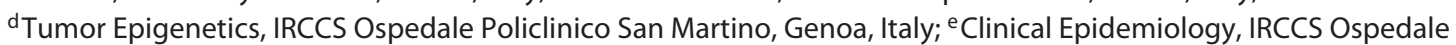 \\ Policlinico San Martino, Genoa, Italy
}

\section{Keywords}

Early disease $\cdot$ Gene expression · Insulin-like growth factors

\begin{abstract}
Introduction: Insulin and the insulin-like growth factor (IGF) family play a key role in breast cancer (BC). Objective: In this study, we evaluated on a genomic scale the potential prognostic value of insulin signaling in early BC. Methods: Candidate genes were selected from the published literature and gene expression profiling experiments. Three publicly available BC datasets, containing gene expression data on 502 cases, were used to test the prognostic ability of the score. The gene signature was developed on GSE1456, containing microarray data from 159 patients, split into a training set (102 breast tumors) and a validation set $(n=57)$. GSE3494 and GSE2990 (350 patients) were used for external validation. Univariate Mann-Whitney test was used to identify genes differentially expressed between relapsed and nonrelapsed patients. Expression of genes significantly correlated with relapse was combined in a linear score. Patients were classified as low or high risk with respect to the median value. Results: On the training set, 15 genes turned out to be differentially expressed: 8-year disease-free survival (DFS) was 51 and $91 \%$ in the high- and low-risk group ( $p<0.001)$, respectively. In the validation set, DFS was 97 and $54 \%$ ( $p=$ 0.009), respectively. External validation: 8-year DFS was 72 and $61 \%$, respectively, in GSE3494 $(p=0.03)$ and 74 and 55\%
\end{abstract}

in GSE2990 ( $p=0.03$ ). By multivariate analyses, the insulin signature was significantly associated with DFS, independently of age, hormone receptor status, nodal status, and grade. Conclusions: Our findings indicate that the insulin pathway is involved in BC prognosis at a genomic level and provide a window of selectivity for preventive and treatment strategies targeting the insulin/IGF pathway in BC patients.

(c) 2020 S. Karger AG, Basel

\section{Background}

In the past 20 years, a substantial body of evidence has developed regarding the role of insulin and the insulinlike growth factor (IGF) family in breast cancer (BC) [1]. This research, mainly focused on the IGF system, led to the development of antibodies targeting the IGF-1 receptor which have been tested in clinical trials, in many types of cancer, with inconsistent results so far [2].

Along with the metabolic effects of insulin on glucose balance [3], insulin has also been shown to induce cancer cell proliferation. The pathways downstream of the insulin/IGF system are well defined: IGF-I and insulin activate the tyrosine kinase growth receptor pathway, that is, the insulin, IGF-I, and hybrid IGF-I/insulin receptors, all of which are overexpressed in BC cells [4]. Activation of these receptors results in upregulation of the insulin receptor substrate-2 (IRS2), leading to downstream activa- 
tion of the MAPKinase and PI3K-Akt pathways [5]. Moreover, insulin may also modulate circulating levels of IGFs and their binding proteins. Epidemiological observations have provided evidence that higher circulating insulin levels are associated with an adverse outcome in early $\mathrm{BC}$ patients $[6,7]$. These data suggest that the insulin pathway itself plays a major role in $\mathrm{BC}$ prognosis and may represent a therapeutic target, especially in those patients exposed to high plasmatic levels. Hyperinsulinemia generally reflects the presence of insulin resistance, a syndrome characterized by decreased insulin sensitivity in peripheral target tissues, such as muscle and fat, which includes abnormal laboratory findings, such as glucose intolerance, impaired lipid metabolism, and signs of a chronic inflammatory state, and manifests as obesity, hypertension, and diabetes $[8,9]$. In nondiabetic women with early $\mathrm{BC}$ it has been observed that hyperinsulinemia is associated with the presence of insulin resistance [10].

In an attempt to clarify the role of insulin in cancer outcome, we hypothesized that it might exert its influence on tumor aggressiveness by modulating gene expression of BC cells. With these premises, the aim of this study was to evaluate the prognostic role of genes related to the insulin and the IGF pathways in early BC by using publicly available gene datasets.

\section{Methods}

\section{Gene Selection}

Candidate genes were selected from the published literature, genomic databases, pathway analysis, and from gene expression profiling experiments performed in peripheral tissues of healthy subjects screened for insulin resistance by the euglycemic insulin clamp technique. In particular, this gene set was identified by microarray profiling (Affymetrix, Santa Clara, CA, USA) on skeletal muscle biopsies of nondiabetic insulin-sensitive and insulin-resistant Pima Indians $[11,12]$. These genes can be functionally categorized into various classes including cell growth, signal transduction, ion transport, transcriptional regulation, protein metabolism, structural genes for the cytoskeleton, lipid and carbohydrate metabolism, and chronic inflammation. Data are deposited in NCBI Gene Expression Omnibus (GEO; available at http://www. ncbi.nlm.nih.gov/geo/, GEO Series accession number GSE2508). In addition, due to the well-known interactions between the insulin and the IGF pathways in cancer cells, genes related to the IGF pathway were selected on Gene Ontology. The panel of selected genes and major pathways is shown in Table 1.

\section{Microarray Datasets}

We used 3 publicly available BC datasets, GSE1456 [13], GSE3494 [14], and GSE2990 [15], including gene expression data on a total of 590 cases with clinical follow-up. The 3 datasets, containing raw intensity data of Affymetrix HU133A arrays, were downloaded and pre-processed using R/Bioconductor (GCRMA package, quantile normalization, median polish summarization [16]). The 3 datasets were pre-processed together using the supercomputer Michelangelo (www.litbio.org). Since the original datasets were found to contain in part the same patients, 88 samples
Table 1. Genes related to the insulin and the insulin growth factor pathway evaluated in this study

\begin{tabular}{|c|c|}
\hline Insulin pathway & $\begin{array}{l}\text { Insulin, insulin-induced genes, insulin-de- } \\
\text { grading enzyme (IDE) } \\
\text { Insulin receptor } \\
\text { Insulin receptor substrate (IR-S1, } \\
\text { IR-S2, IR-S4) } \\
\text { Similar to IR-S-like protein } \\
\text { Insulin-like factors } \\
\text { Insulin promoter factor } 1 \\
\text { Homeodomain transcription factor }\end{array}$ \\
\hline $\begin{array}{l}\text { Glucose } \\
\text { metabolism }\end{array}$ & $\begin{array}{l}\text { Solute carrier family 2: GLUT1, GLUT4 } \\
\text { Hydroxysteroid (11- } \beta \text { ) dehydrogenase } 1 \\
\text { CD68 antigen (macrophage marker) }\end{array}$ \\
\hline Inflammation & $\begin{array}{l}\text { IL-6 } \text { (interferon, } \beta 2 \text { ) } \\
\text { IL- } 6 \text { signal transducer } \\
\text { IL-6 receptor } \\
\text { TNF superfamily, member } 2\end{array}$ \\
\hline IGF pathway & $\begin{array}{l}\text { IGF } 1 \text { (somatomedin C) } \\
\text { IGF } 2 \text { (somatomedin A) } \\
\text { IGF BP (binding proteins) } \\
\text { IGF-II mRNA-BP2 } \\
\text { IGF-II mRNA-BP } 3 \\
\text { IGF } 1 \text { receptor } \\
\text { IGF } 2 \text { receptor } \\
\text { IGF } 2 \text { antisense } \\
\text { IGF II associated protein }\end{array}$ \\
\hline
\end{tabular}

present in GSE3494 and in GSE2990 were removed from the latter. This procedure resulted in a total of 502 cases suitable for the present study. Table 2 describes the characteristics of the 3 datasets.

\section{Statistical Methods}

The insulin gene signature was developed on GSE1456. The dataset was split by a random procedure into a training and a validation set (ratio 2:1). The signature was developed on the training set. Once the signature had been fully specified, the validation set was accessed once and only for estimating the prediction accuracy of the identified genes. The prognostic value of the signature was tested on the validation set by Kaplan-Meier survival analysis and Cox regression analysis. A multivariate Cox model was run on the whole dataset adjusting for molecular subtype. An external validation was performed on GSE3494, containing 249 early BC patients, and on GSE2990, containing 101 patients, by Kaplan-Meier survival analysis and Cox regression analysis on both datasets (GSE3494 and GSE2990). A stratified multivariate Cox model was run on the merged GSE3494/GSE2990 dataset adjusting for age, tumor size, nodal status, hormone receptor status, and tumor grade. The final model was obtained by means of a background procedure based on the likelihood ratio test. To verify the independence of insulin/IGF signature, we performed a comparison with the 21-Recurrence Score, a clinically validated assay, based on expression of 21 genes in women with $\mathrm{ER}+$, lymph node-negative BC treated with adjuvant tamoxifen [17].

\section{Insulin Gene Signature Development}

A univariate Cox regression analysis was run to select genes whose expression levels were significantly correlated with diseasefree survival (DFS). When multiple probes were mapped on the 
Table 2. Characteristics of the gene expression datasets included in this study

\begin{tabular}{llll}
\hline Dataset & GEO: GSE1456 & GEO: GSE3494 & GEO: GSE2990 \\
\hline Patients, $n$ & 159 & 249 & 101 \\
Time of sample collection & $1994-1996$ & $1987-1989$ & $1993-1995$ \\
Institution & Karolinska Institutet, & Karolinksa Institutet, & John Radcliffe Hospital, \\
& Stockholm, Sweden & Uppsala, Sweden & Oxford, UK \\
Mean age, years & 58 & 62 & 58 \\
Mean tumor size, mm & 22 & 22 & 22 \\
Tumor size $<21$ mm, $\%$ & 62 & 51 & 58 \\
Grade 3, $n(\%)$ & $65(41)$ & $196(78)$ & $31(31)$ \\
ER positive, $n(\%)$ & $130(82)$ & $222(89)$ & $85(84)$ \\
Positive lymph nodes, $n(\%)$ & $60(38)$ & $77(31)$ & $15(15)$ \\
Adjuvant HT, $n(\%)$ & $114(72)$ & $67(27)$ & 0 \\
Adjuvant chemotherapy, $n(\%)$ & $30(19)$ & $60(24)$ & 7.0 \\
Mean follow-up, years & 6.2 & 7.1 & 40 \\
Proportion relapsed, $\%$ & 25 & 36 & Affymetrix \\
Platform & Affymetrix & Affymetrix & \\
\hline
\end{tabular}

HT, hormone therapy.

Table 3. List of the 15 genes of the insulin gene score

\begin{tabular}{|c|c|c|c|c|c|c|c|}
\hline \multirow[t]{2}{*}{ Probe set ID } & \multirow[t]{2}{*}{ Gene symbol } & \multicolumn{2}{|c|}{ Low risk } & \multicolumn{2}{|c|}{ High risk } & \multirow[t]{2}{*}{$p$ value } & \multirow{2}{*}{$\begin{array}{l}\text { Score } \\
\text { coefficient }\end{array}$} \\
\hline & & mean & SD & mean & SD & & \\
\hline X201627_s_at & INSIG1 & 6.9 & 1.3 & 7.7 & 0.8 & 0.004 & 0.31 \\
\hline X203328_x_at & IDE & 2.9 & 1.0 & 3.6 & 1.5 & 0.005 & 0.34 \\
\hline X204686_at & IRS1 & 8.0 & 1.4 & 7.2 & 1.9 & 0.01 & -0.17 \\
\hline X209184_s_at & IRS2 & 5.7 & 1.7 & 4.8 & 1.9 & 0.03 & 0.22 \\
\hline X204863_s_at & IL6 & 8.3 & 1.8 & 6.8 & 2.7 & 0.001 & 0.02 \\
\hline X209295_at & TNF R10b & 7.3 & 1.0 & 6.8 & 1.2 & 0.04 & -0.03 \\
\hline X218368_s_at & TNF R12a & 7.4 & 1.1 & 7.9 & 0.8 & 0.03 & 0.28 \\
\hline X214581_x_at & TNF R21 & 3.8 & 1.0 & 4.7 & 1.5 & 0.002 & 0.11 \\
\hline X214329_x_at & TNF 10 & 8.6 & 1.5 & 7.8 & 1.8 & 0.04 & -0.07 \\
\hline X209540_at & IGF1 & 7.0 & 2.1 & 4.8 & 2.1 & $<0.001$ & -0.44 \\
\hline X202410_x_at & IGF2 & 5.1 & 2.4 & 3.8 & 2.1 & 0.02 & 0.09 \\
\hline X203628_at & IGF1R & 8.9 & 2.2 & 7.8 & 3.0 & 0.02 & 0.05 \\
\hline X201508_at & IGFBP4 & 10.0 & 1.2 & 9.5 & 1.2 & 0.05 & 0.05 \\
\hline X203851_at & IGFBP6 & 6.3 & 1.6 & 4.8 & 1.9 & $<0.001$ & -0.34 \\
\hline X201163_s_at & IGFBP7 & 12.4 & 0.5 & 12.2 & 0.7 & 0.04 & 1.37 \\
\hline
\end{tabular}

same gene ID, the most correlated one by univariate analysis was chosen. Those genes related to DFS with a significance level $<0.05$ were included in a multivariate Cox regression model. Their expression levels were combined in a weighted linear score:

$$
\text { InsulinGeneScore }=\sum_{i} w_{i} \text { value }_{i}
$$

where $w_{i}$ are weights determined by the coefficients of the multivariate Cox regression model and value $_{i}$ are the gene expression levels. The median value of the score was chosen as the cutoff to classify patients at low or high risk of disease relapse.

\section{Results}

One hundred forty-three probe sets were identified on the Affymetrix chip, corresponding to 85 insulin-related genes. The genes were tested on the GSE1456 dataset. This dataset contains raw data from 159 early BC patients with a median follow-up of 6.1 years. The dataset was split by a random procedure into a training set and a validation set of 102 and 57 primary breast tumors, respectively. On the training set, 15 genes turned out to be differentially expressed between relapsed and nonrelapsed patients with a significance level $<0.05$. The insulin signature con- 
Fig. 1. Individual HRs related to the overexpression of the 15 genes.

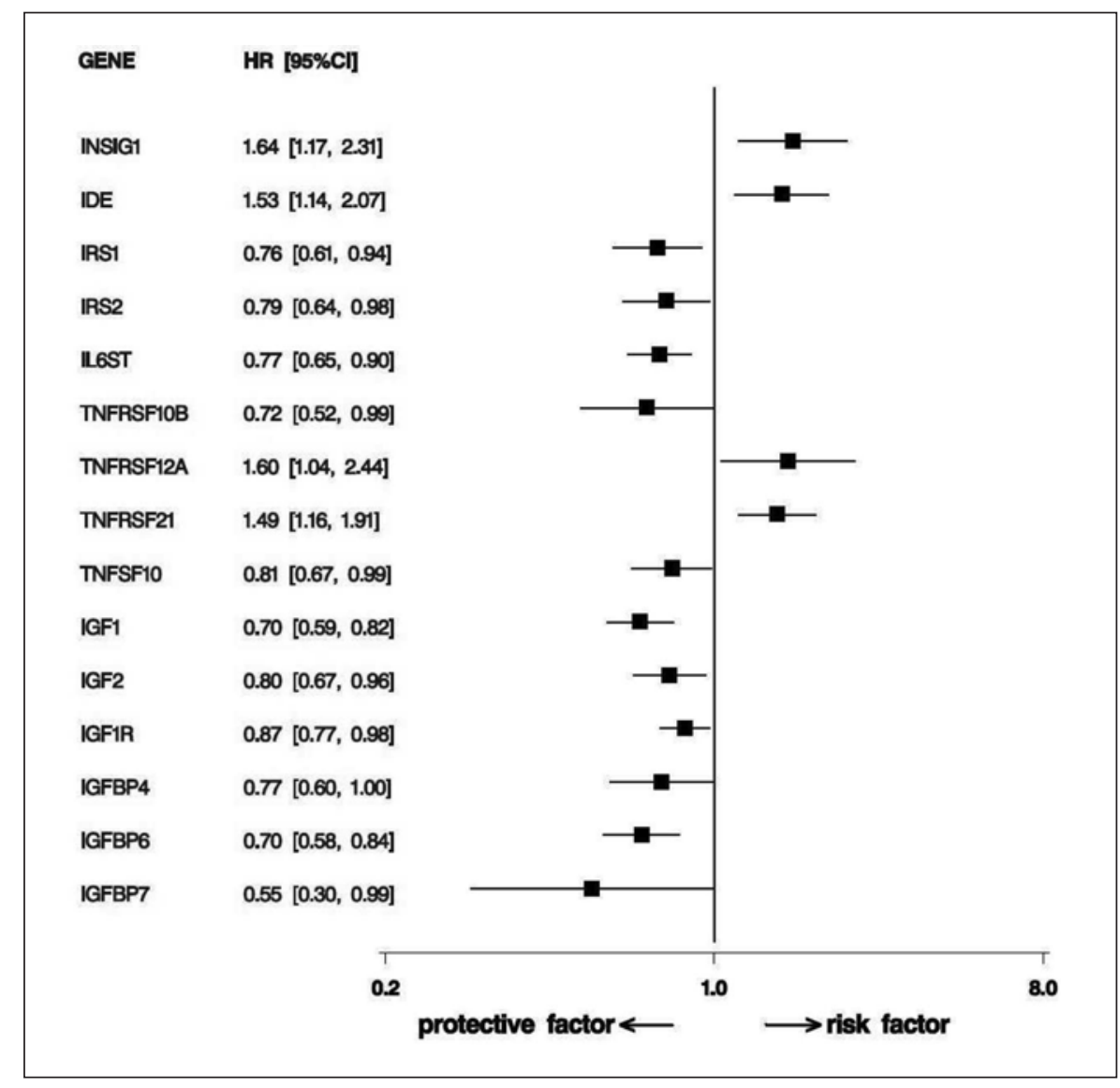

TRAINING SET

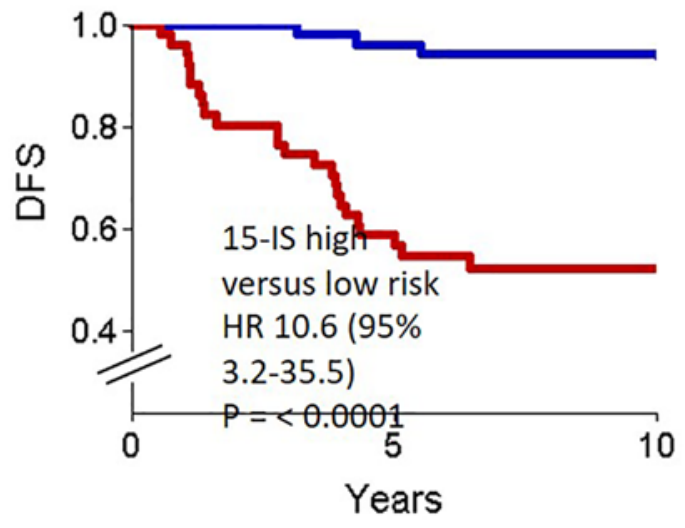

VALIDATION SET

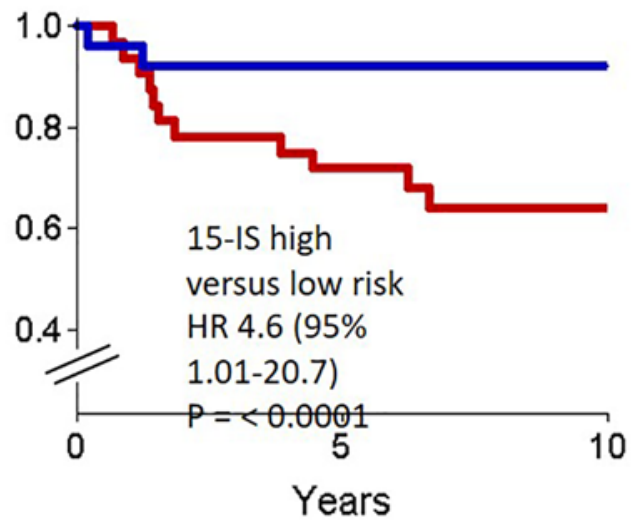

Fig. 2. Kaplan-Meier curves of DFS in GSE1456. DFS, disease-free survival; 15-IS, 15-Insulin Sensitivity.

sists of a score, defined as the linear combination of the 15 genes with the standardized Cox's regression coefficient as the weight (Table 3). The median value of the score was used to classify patients as high and low risk.

The 15 genes can be functionally divided into 3 classes: those belonging to the insulin pathway ( 4 genes, $27 \%$ ), the chronic inflammation pathway (5 genes, 30\%), and the IGF pathway (6 genes, $40 \%$ ). Individual hazard ratios (HRs) related to the overexpression of each of the 15 genes are presented in Figure 1. In the training set (102 patients), the 8-year DFS was 91\% (standard error [SE] 4\%) in the low-risk group and 51\% (SE 8\%) in the high-risk group 


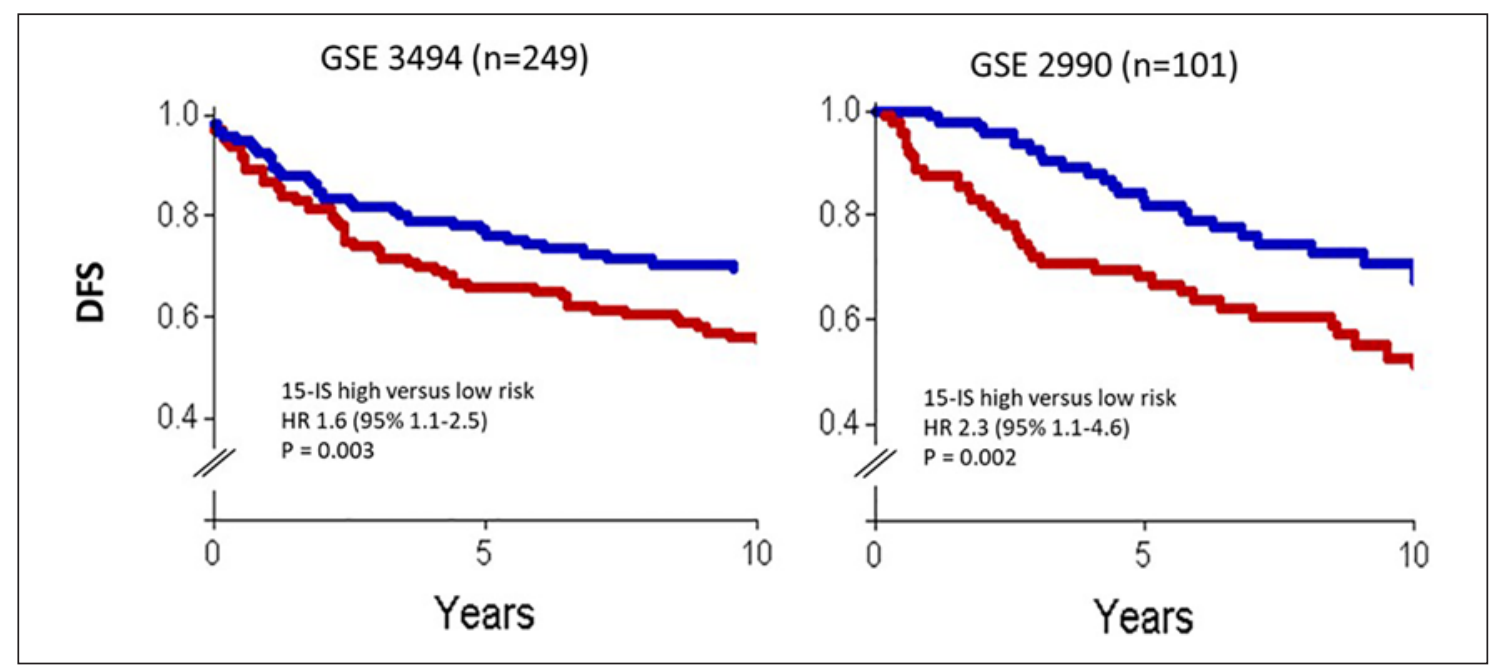

Fig. 3. Kaplan-Meier curves of DFS in the 2 datasets used for external validation. DFS, disease-free survival; 15IS, 15-Insulin Sensitivity.

$(p<0.001)$. Univariate DFS HR (high vs. low risk) was 10.6 (95\% CI 3.2-35.5, $p<0.0001$ ). The gene signature was then tested on the 57 patients included in the validation set. The 8-year DFS was 97\% (SE 3\%) and 54\% (SE 10\%) in the low-risk and the high-risk group $(p=0.03)$, respectively, supporting the discriminating ability of the identified insulin sensitivity score. Univariate DFS HR was 4.6 (95\% CI 1.01-20.7, $p=0.04$ ). Figure 2 shows Kaplan-Meier survival curves according to the high- and low-risk group in the training and validation set.

The identified insulin signature was then tested on 2 external independent datasets, GSE3494 including 249 early BC patients and GSE2990 including 101 early BC patients. The score significantly predicted DFS in both datasets: in GSE3494, the 8-year DFS was 72\% (SE 5\%) in the low-risk group and 61\% (SE 4\%) in the high-risk group $(p=0.04)$. By univariate analysis, the DFS HR was 1.6 (95\% CI 1.1-2.5, $p=0.03$ ). In GSE2990, the 8-year DFS was $74 \%$ (SE 7\%) and 55\% (SE 8\%) in the low-risk and the high-risk group, respectively $(p=0.008)$. By univariate analysis, the DFS HR was 2.3 (95\% CI 1.1-4.6, $p=$ 0.02 ). Kaplan-Meier survival curves obtained in the 2 independent datasets are shown in Figure 3.

By multivariate analysis of the merged datasets (GSE3494 and GSE2990), the insulin signature turned out to be significantly associated with DFS (HR 1.5, 95\% CI 1.0-2.3, $p=0.04$ ), independently of other established prognostic factors, including age, ER status, nodal status and grade, except for tumor size (Table 4).

Three hundred fifty-seven patients (249 from GSE3494 and 108 from GSE2990) were assessable for type of adjuvant therapy. Of these, $130(30 \%)$ received endocrine therapy and 41 (9\%) received chemotherapy according to local clinical practice. The test for interaction between
Table 4. Multivariate Cox analysis of GSE3494 and GSE2990 merged dataset (350 patients)

\begin{tabular}{lcc}
\hline & HR $(95 \% \mathrm{CI})$ & $p$ value \\
\hline Age & & \\
$\quad \leq 50$ years & 1 (ref.) & 0.07 \\
$\quad>50$ years & $0.7(0.4-1.0)$ & \\
Tumor size & 1 (ref.) & \\
$\quad \leq 2 \mathrm{~cm}$ & $2.3(1.5-3.5)$ & \\
$>2 \mathrm{~cm}$ & & \\
ER status & 1 (ref.) & \\
$\quad$ Negative & $1.0(0.6-1.6)$ & \\
$\quad$ Positive & 1 (ref.) & 0.1 \\
Nodes & $1.4(0.9-2.1)$ & \\
$\quad$ Negative & & \\
$\quad$ Positive & 1 (ref.) & \\
Grade & $1.2(0.7-2.0)$ & 0.9 \\
1 & $1.0(0.5-1.8)$ & \\
2 & 1 (ref.) & \\
3 & $1.5(1.0-2.3)$ & 0.04 \\
Insulin gene score & & \\
Low & & \\
High & &
\end{tabular}

type of adjuvant systemic therapy (yes or no) and the insulin signature was statistically significant $(p=0.009)$. In patients who received adjuvant systemic therapy, either endocrine therapy or chemotherapy, the insulin signature identified a subset of patients with high risk of relapse despite treatment (HR 2.77, 95\% 1.59-4.82, $p<$ 0.0001).

Finally, we performed a comparison of the insulin score with the 21-Recurrence Score (21-gene signature) in the validation set (57 patients); a positive correlation between 2 signatures was observed $(r=0.34, p<0.05$; Fig- 


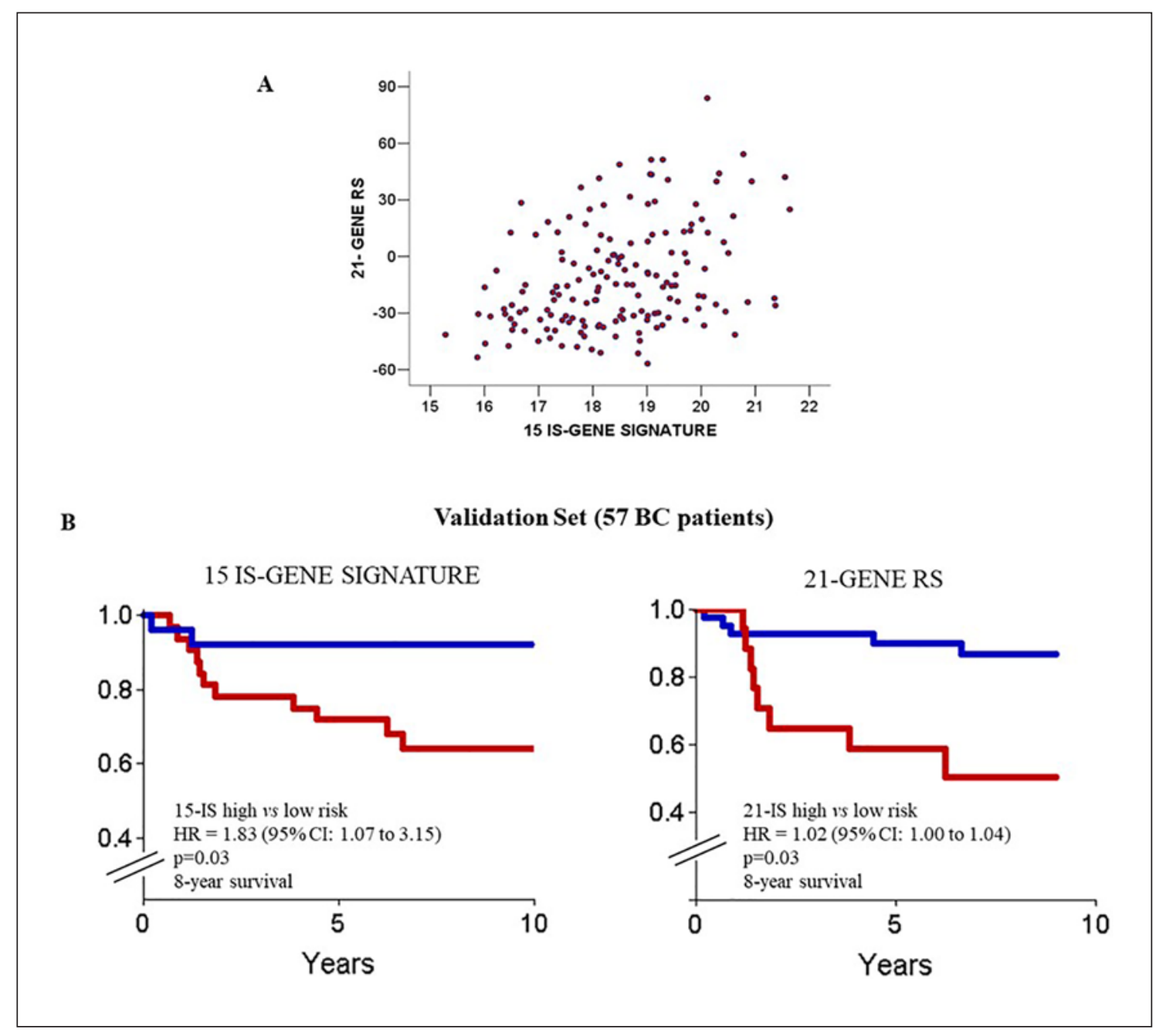

Fig. 4. Scatter plot (A) and Kaplan-Meier curves (B) of DFS in comparison with 15-IS gene and 21-RS signatures. DFS, disease-free survival; 15-IS, 15-Insulin Sensitivity; 21-RS, 21-Recurrence Score.

ure $4 \mathrm{a}$ ). When using the median value of the score to classify patients as high and low risk in a multivariate Cox analysis, HR (high vs. low risk) for DFS was 1.83 (95\% CI $1.07-3.15, p=0.03$ ) for 15-Insulin Sensitivity signature, while it was 1.02 (95\% CI 1.00-1.04, $p=0.03$ ) in 21-Recurrence Score. This observation supports the discriminating ability of the identified insulin sensitivity score and its independence from the 21-Recurrence Score (Fig. 4b).

\section{Discussion}

In this study, we used a mechanism-driven approach to evaluate the involvement of insulin signaling in $\mathrm{BC}$ prognosis on a genomic scale. In particular, we identified a gene signature based on the differential expression of 15 genes related to the insulin pathway that was strongly associated with DFS in early BC. The ability of the insulin signature to predict outcome was maintained after external validation performed on 2 different large independent datasets. In Cox's multivariate analyses, the insulin gene signature was found to be an independent variable in predicting DFS when molecular subtype and other established factors, such as nodal status, histologic grade, hormone receptor status, and age, were taken into account.

The genes included in the insulin signature showed no overlap with other prognostic classifiers, with the exception of the IGF1 gene, shared with the prognostic signature developed by Pawitan et al. [13]. However, this last observation, due to the nature of this analysis, has to be considered as exploratory, since no selection of ER-positive samples was done.

The 15 genes can be functionally divided into 3 classes: those belonging to the insulin pathway (27\%), the chronic inflammation pathway (30\%), and the IGF pathway (40\%). The insulin pathway is present with 4 genes: INSIG1, IDE, IRS1, and IRS2. Preclinical evidence indicates 
that INSIG1 (insulin-induced gene 1) is involved in regulation of transcription and is upregulated by exposure to genotoxic agents in cancer models [18]; IDE (insulin-degrading enzyme) is a zinc metalloprotease involved in intracellular degradation of insulin recently detected in malignant tissues, including BC [19]. IRS1 and IRS2 (insulin receptor substrate 1 and 2) are major substrates for the insulin and the IGF receptors and they mediate signals to promote tumor cell survival, growth, and motility in BC cells [20]. Five genes (IL-6, TNFR10b, TNFR12a, TNFR21, and TNF10), pertinent to the chronic inflammation pathway, turned out to be differentially expressed between relapsed and nonrelapsed patients; among these, IL-6 (interleukin-6) and TNF (tumor necrosis factor) are multifunctional cytokines produced by a number of tumor cells, including BC $[21,22]$, and are correlated with an adverse prognosis in advanced BC stages [23].

In the IGF-related group, high expression of the IGF1 gene was associated with the low risk profile. These data are consistent with available evidence indicating that at a cellular level, high IGF1 expression is correlated with a more favorable outcome in BC patients. Among the other IGF-related genes, IGF-binding protein genes, namely IGFBP4, IGFBP6, and IGFBP7, were also overexpressed in the low-risk profile. IGFBP4 has been shown to be an independent prognostic factor in $\mathrm{BC}$ and a predictor of endocrine responsiveness [24]. Finally, both up- and downregulation of IGFBP7 have been reported in BC [25] and are involved in modulating VEGF expression and signaling as well [26].

These data suggest that insulin signaling may be a key regulatory pathway in $\mathrm{BC}$ and may represent a therapeutic target. The implementation of therapies that target hyperinsulinemia is one of the most intriguing and emerging fields of research in different types of tumors. In addition to lifestyle interventions that are effective in reducing insulin levels in BC survivors [27], but strongly dependent on patient attitude and compliance, interest has focused on metformin, an anti-diabetic drug widely prescribed for the treatment of hyperglycemia and hyperinsulinemia. Cheap and generally well tolerated [28], meftormin has also been shown to retain antiproliferative properties, through the activation of the AMPK pathway, in preclinical BC models [29]. Epidemiologic evidence also indicated that in diabetic patients, metformin is associated with reduced risk of cancer incidence and mortality [30]. Moreover, in a series of 2,529 BC patients receiving preoperative chemotherapy at MD Anderson Cancer Center, the probability of achieving a pathological complete response was reported to be $30 \%$ higher in diabetic patients receiving metformin as compared to nondiabetic patients [31].

In the past few years, these data prompted the exploitation of metformin as an anticancer drug in $\mathrm{BC}$, with inconsistent results. Our group performed 2 randomized clinical trials in early and advanced $\mathrm{BC}$ patients. In the first one, 200 nondiabetic patients with operable BC were randomized to receive metformin or placebo in a window-of-opportunity, preoperative study. The primary endpoint was tumor proliferation. Overall, metformin did not significantly impact Ki-67; however, a significant difference was detected according to the level of insulin sensitivity [32].

In the MYME study [33], 122 nondiabetic women with HER2-negative metastatic breast cancer were randomized to receive first-line chemotherapy plus metformin or chemotherapy alone; the final results failed to provide evidence of an additional benefit of metformin in terms of progression-free and overall survival. Noteworthy, a significantly worse outcome was observed in insulin-resistant patients (HOMA $\geq 2.59$, independent of metformin administration). A biological collateral study, TransMYME, evaluated in the same patients the prognostic role of IGF-1R expression on circulating tumor cells, indicating that loss of IGF-1R expression was associated with a significantly worse outcome [34]. Yet, a possible limitation of this study was the absence of any information related to individual patient metabolic status, including body mass index, level of insulin sensitivity, and nutritional status.

In conclusion, our study suggests that it is possible to identify a subset of $\mathrm{BC}$ patients whose prognosis is strongly modulated by a set of genes related to the insulin pathway. This might help to better individualize lifestyle and therapeutic interventions targeting insulin signaling, by selecting those patients at high risk of relapse according to the insulin signature and to evaluate the prognostic impact of epigenetic modulations induced by these types of interventions.

\section{Acknowledgment}

Lega Italiana per la Lotta Contro i Tumori (LILT) and Italian Association for Cancer Research (AIRC).

\section{Statement of Ethics}

This study is based on data from publicly available gene datasets; ethical approval was not required.

\section{Conflict of Interest Statement}

A.G. received honoraria as expert of testimony and speaker bureau from EISAI, Roche, Lilly, Novartis, Pfizer, TEVA, Astra Zeneca, Celgene and Daichii Sankyo. The remaining authors have no conflicts of interest to declare. 


\section{Funding Sources}

Funded by AGING Project - Department of Excellence - DIMET, UPO, Novara, Italy (AG) and AIRC, Italian Association for Cancer Research (AG).

\section{Author Contributions}

A.G., P.B., and U.P. conceived and designed the study. M.S., M.P., V.M., and A.A. downloaded and performed all the analyses on the datasets. A.G., M.S., M.P., V.M., and U.P. wrote the research article. A.G., P.B., M.S., M.P., A.A., V.M., and U.P. interpreted the results and revised the manuscript. All authors contributed to data analysis, drafting, and revising the article and gave final approval of the version to be published.

\section{References}

1 Yee D. Targeting insulin-like growth factor pathways. Br J Cancer. 2006 Feb;94(4):465-8.

2 Beckwith $\mathrm{H}$, Yee D. Minireview: Were the IGF Signaling Inhibitors All Bad? Mol Endocrinol. 2015 Nov;29(11):1549-57.

3 Scheen AJ, Lefèbvre PJ. Insulin action in man. Diabetes Metab. 1996 Apr;22(2):105-10.

4 Pollak M. Insulin and insulin-like growth factor signalling in neoplasia. Nat Rev Cancer. 2008 Dec;8(12):915-28.

5 Sandhu MS, Dunger DB, Giovannucci EL. Insulin, insulin-like growth factor-I (IGF-I), IGF binding proteins, their biologic interactions, and colorectal cancer. J Natl Cancer Inst. 2002 Jul;94(13):972-80.

6 Goodwin PJ, Ennis M, Pritchard KI, Trudeau ME, Koo J, Madarnas Y, et al. Fasting insulin and outcome in early-stage breast cancer: results of a prospective cohort study. J Clin Oncol. 2002 Jan;20(1):42-51.

7 Borugian MJ, Sheps SB, Kim-Sing C, Van Patten C, Potter JD, Dunn B, et al. Insulin, macronutrient intake, and physical activity: are potential indicators of insulin resistance associated with mortality from breast cancer? Cancer Epidemiol Biomarkers Prev. 2004 Jul; 13(7):1163-72.

8 Ferrannini E. Is insulin resistance the cause of the metabolic syndrome? Ann Med. 2006; 38(1):42-51.

9 Bonora E, Capaldo B, Perin PC, Del Prato S, De Mattia G, Frittitta L, et al.; Group of Italian Scientists of Insulin Resistance (GISIR). Hyperinsulinemia and insulin resistance are independently associated with plasma lipids, uric acid and blood pressure in non-diabetic subjects. The GISIR database. Nutr Metab Cardiovasc Dis. 2008 Nov; 18(9):624-31.

10 Goodwin PJ, Ennis M, Bahl M, Fantus IG, Pritchard KI, Trudeau ME, et al. High insulin levels in newly diagnosed breast cancer patients reflect underlying insulin resistance and are associated with components of the insulin resistance syndrome. Breast Cancer Res Treat. 2009 Apr; 114(3):517-25.

11 Yang X, Pratley RE, Tokraks S, Bogardus C, Permana PA. Microarray profiling of skeletal muscle tissues from equally obese, non-diabetic insulin-sensitive and insulin-resistant Pima Indians. Diabetologia. 2002 Nov;45(11): 1584-93.

12 Lee YH, Tokraks S, Pratley RE, Bogardus C, Permana PA. Identification of differentially expressed genes in skeletal muscle of non-diabetic insulin-resistant and insulin-sensitive Pima Indians by differential display PCR. Diabetologia. 2003 Nov;46(11):1567-75.
13 Pawitan Y, Bjöhle J, Amler L, Borg AL, Egyhazi S, Hall P, et al. Gene expression profiling spares early breast cancer patients from adjuvant therapy: derived and validated in two population-based cohorts. Breast Cancer Res. 2005;7(6):R953-64.

14 Miller LD, Smeds J, George J, Vega VB, Vergara L, Ploner A, et al. An expression signature for p53 status in human breast cancer predicts mutation status, transcriptional effects, and patient survival. Proc Natl Acad Sci USA. 2005 Sep;102(38):13550-5.

15 Sotiriou C, Wirapati P, Loi S, Harris A, Fox S, Smeds J, et al. Gene expression profiling in breast cancer: understanding the molecular basis of histologic grade to improve prognosis. J Natl Cancer Inst. 2006 Feb;98(4):262-72.

16 Irizarry RA, Bolstad BM, Collin F, Cope LM, Hobbs B, Speed TP. Summaries of Affymetrix GeneChip probe level data. Nucleic Acids Res. 2003 Feb;31(4):e15.

17 Paik S, Shak S, Tang G, Kim C, Baker J, Cro$\operatorname{nin} \mathrm{M}$, et al. A multigene assay to predict recurrence of tamoxifen-treated, node-negative breast cancer. N Engl J Med. 2004 Dec; 351(27):2817-26.

18 Simões ML, Hockley SL, Schwerdtle T, Gamboa da Costa G, Schmeiser HH, Phillips DH, et al. Gene expression profiles modulated by the human carcinogen aristolochic acid I in human cancer cells and their dependence on TP53. Toxicol Appl Pharmacol. 2008 Oct; 232(1):86-98.

19 Yfanti C, Mengele K, Gkazepis A, Weirich G, Giersig C, Kuo WL, et al. Expression of metalloprotease insulin-degrading enzyme insulysin in normal and malignant human tissues. Int J Mol Med. 2008 Oct;22(4):421-31.

20 Gibson SL, Ma Z, Shaw LM. Divergent roles for IRS- 1 and IRS- 2 in breast cancer metastasis. Cell Cycle. 2007 Mar;6(6):631-7.

21 Knüpfer H, Preiss R. Significance of interleukin-6 (IL-6) in breast cancer (review). Breast Cancer Res Treat. 2007 Apr;102(2):129-35.

22 Hodge DR, Hurt EM, Farrar WL. The role of IL- 6 and STAT3 in inflammation and cancer. Eur J Cancer. 2005 Nov;41(16):2502-12.

23 Sansone P, Storci G, Tavolari S, Guarnieri T, Giovannini C, Taffurelli M, et al. IL-6 triggers malignant features in mammospheres from human ductal breast carcinoma and normal mammary gland. J Clin Invest. 2007 Dec; 117(12):3988-4002.

24 Zhu Y, Wang A, Liu MC, Zwart A, Lee RY, Gallagher A, et al. Estrogen receptor alpha positive breast tumors and breast cancer cell lines share similarities in their transcriptome data structures. Int J Oncol. 2006 Dec;29(6): 1581-9.
25 Seth A, Kitching R, Landberg G, Xu J, Zubovits J, Burger AM. Gene expression profiling of ductal carcinomas in situ and invasive breast tumors. Anticancer Res. 2003 MayJun;23 3A:2043-51.

26 Tamura K, Hashimoto K, Suzuki K, Yoshie $M$, Kutsukake $M$, Sakurai T. Insulin-like growth factor binding protein-7 (IGFBP7) blocks vascular endothelial cell growth factor (VEGF)-induced angiogenesis in human vascular endothelial cells. Eur J Pharmacol. 2009 May;610(1-3):61-7.

27 Ligibel JA, Campbell N, Partridge A, Chen WY, Salinardi T, Chen H, et al. Impact of a mixed strength and endurance exercise intervention on insulin levels in breast cancer survivors. J Clin Oncol. 2008 Feb;26(6):907-12.

28 Scarpello JH, Howlett HC. Metformin therapy and clinical uses. Diab Vasc Dis Res. 2008 Sep;5(3):157-67.

29 Zakikhani M, Dowling R, Fantus IG, Sonenberg N, Pollak M. Metformin is an AMP kinase-dependent growth inhibitor for breast cancer cells. Cancer Res. 2006 Nov;66(21): 10269-73.

30 Bowker SL, Majumdar SR, Veugelers P, Johnson JA. Increased cancer-related mortality for patients with type 2 diabetes who use sulfonylureas or insulin. Diabetes Care. 2006 Feb; 29(2):254-8.

31 Jiralerspong S, Palla SL, Giordano SH, MericBernstam F, Liedtke C, Barnett CM, et al. Metformin and pathologic complete responses to neoadjuvant chemotherapy in diabetic patients with breast cancer. J Clin Oncol. 2009 Jul;27(20):3297-302.

32 Bonanni B, Puntoni M, Cazzaniga M, Pruneri G, Serrano D, Guerrieri-Gonzaga A, et al. Dual effect of metformin on breast cancer proliferation in a randomized presurgical trial. J Clin Oncol. 2012 Jul;30(21):2593-600.

33 Nanni O, Amadori D, De Censi A, Rocca A, Freschi A, Bologna A, et al.; MYME investigators. Metformin plus chemotherapy versus chemotherapy alone in the first-line treatment of HER2-negative metastatic breast cancer. The MYME randomized, phase 2 clinical trial. Breast Cancer Res Treat. 2019 Apr; 174(2):433-42.

34 Gennari A, Foca F, Zamarchi R, Rocca A, Amadori D, De Censi A, et al. Insulin-like growth factor-1 receptor (IGF-1R) expression on circulating tumor cells (CTCs) and metastatic breast cancer outcome: results from the TransMYME trial. Breast Cancer Res Treat. 2020 May;181(1):61-8. 